\title{
The basics of economic evaluation in mental healthcare
}

\author{
James Shearer \& Sarah Byford
}

\begin{abstract}
SUMMARY
Economic evaluation involves the comparative analysis of the costs and consequences of alternative (different) treatment options. Economic evaluations provide decision makers with information about the relative value for money, or cost-effectiveness, of various treatment programmes. The relative cost-effectiveness of new interventions is a key consideration in health technology assessments by the UK's National Institute for Health and Care Excellence, and economic evaluations alongside randomised controlled trials are routinely requested by funders such as the National Institute for Health Research. This article outlines some of the key concepts and issues in the economic evaluation of mental healthcare.

\section{LEARNING OBJECTIVES}

- Understand the role of economic evaluation in providing information for decision makers

- Understand how economic evaluations are constructed

- Be able to interpret the results of economic evaluations

\section{DECLARATION OF INTEREST}

None
\end{abstract}

The economic impact of mental health problems in the UK is profound and pervasive. Mental illness accounts for up to $23 \%$ of the burden of ill health in the UK and is the largest single cause of disability (Fineberg 2013). This contributes to considerable economic costs, including the cost of National Health Service (NHS) treatment, social care and lost productivity. Mental health is the largest category in the NHS budget, accounting for $£ 10.4$ billion, or $10.8 \%$ of total expenditure in 2008-2009 (Fineberg 2013). Other services that respond to mental health problems in the community include health and social care services provided by local authorities and nonstatutory sector organisations, and interventions by the criminal justice system. In addition, mental health problems can place a substantial burden on unpaid informal carers, including family members and friends. The total cost of all of these resources was estimated at $£ 22.5$ billion in 2007 , with the NHS contributing about one-third or $£ 8.4$ billion (McCrone 2008).

The growth in expenditure on mental healthcare has been driven by demographic changes (e.g. ageing populations), technological changes (e.g. new medications, new therapies, new diagnoses) and changing expectations (e.g. less stigma, more awareness). However, the NHS, local authorities, the criminal justice sector and the third sector (charities, profit and not-for-profit organisations) who are responsible for providing the resources to meet these increasing demands currently face either static or declining funding after inflation. They also face regular political demands to achieve efficiency savings (e.g. delivering the same services at lower cost), with the most recent target for the NHS set at $£ 20$ billion by 2014-2015 (Jacobs 2014).

Health economics examines how individuals, the health system and society confront the reality that healthcare resources are limited, whereas the competing uses for these resources are unlimited. Scarcity means that choices must be made as to which health services should be provided, how they should be provided, how much should be provided and how they should be distributed. An economic evaluation explores these questions through a comparative analysis of the costs and consequences of the unavoidable choices between alternative interventions. The aim of this article is to provide readers with a practical guide to the key concepts and terms used in the economic evaluation of mental health programmes and interventions.

\section{Economics in mental healthcare}

Economics is the study of the production, consumption and distribution of goods and services in a society, and the primary question is how best to allocate these resources among the many competing demands for them (Robinson 1993). In mental health, scarce resources mean that at any one time, there are only so many psychiatrists, psychologists and other mental health professionals working in the healthcare system, there are only so many in-patient beds
James Shearer is a lecturer in health economics at King's Health Economics, King's College London. He has a specific interest in new methods in trial-based economic evaluations, including mental health and the addictions. Sarah Byford is Professor of Health Economics and Director of King's Health Economics. Her primary research interest is in the economic evaluation of mental health and social care services, including services for children and adolescents. Correspondence Dr James Shearer, King's Health Economics, King's College London, Box P024, David Goldberg Centre, De Crespigny Park, London SE5 8AF, UK. Email: james.shearer@kcl.ac.uk 
available on a limited number of psychiatric wards, and there is a limited budget for psychotropic medications and other therapeutic services. Choosing to use these scarce resources in one way will always mean giving up the opportunity to use them in other beneficial ways. Thus, for economists the true cost of choosing one course of action is not its financial cost, but instead it is the value of the benefits that could have been achieved from the next best alternative use of the same resources. Economists call this sacrifice 'opportunity cost'. The opportunity cost of a session of psychotherapy delivered by a clinical psychologist is the benefit foregone of the psychologist doing something else with that time, for example running a group treatment, seeing a different patient or supervising junior colleagues. Every decision to fund a service or treat a patient in a resource-constrained health system entails a loss elsewhere. It is this loss, or opportunity cost, that is the key to understanding the economic perspective (Byford 2010).

The economic criterion for deciding on a desirable allocation of resources is efficiency. In general terms, an efficient allocation of resources occurs when the benefits of a service are maximised given the resources available or, equivalently, by minimising the resources needed to achieve the desired level of benefit (Guiness 2011). Improvements in total benefits gained from resources can be realised by reallocating resources. The key to understanding efficiency is to understand how to define and measure the benefit that we want to maximise and for whom. These important issues will be considered in more detail in our discussion of economic evaluation.

The efficiency criterion aims to ensure that total population health is increased when resources are reallocated, but makes no judgement about which members of society benefit from this increase. However, society may prefer to fund a less efficient service if it believes that service will produce a more equitable distribution of resources. Thus, when undertaking an economic evaluation, which is primarily concerned with efficiency, decision makers need also to consider the equity implications of their choices (Byford 2010).

\section{Economic evaluation}

Economic evaluation has been neatly defined as the 'comparative analysis of alternative courses of action in terms of both their costs and consequences' (Drummond 2005), so it must include consideration of both costs and consequences and involve a comparison between two or more options. These features are essential to the primary objective of an economic evaluation, which is to provide the information about the relative value for money of alternative treatment programmes needed to support funding and priority-setting decisions. Economic evaluations can be conducted in randomised controlled trials (RCTs) using patient-level costs and outcomes (Petrou 2011a), or through decision analytic modelling, which synthesises cost and outcome data from multiple sources (Briggs 2006). The basic task in an economic evaluation is the systematicidentification, measurement, valuation and comparison of all relevant costs and consequences of the alternative interventions under consideration.

\section{Comparators}

Since economics is concerned with the use of scarce resources, implying that the provision of one service must come at the expense of others, economic analysis by definition should involve comparisons between alternative courses of action. The choice of comparison (or control) group can have significant implications for the design of a study and, ultimately, the cost-effectiveness of the interventions under investigation. The less effective the control intervention, the more effective the alternative will appear in comparison, and the greater the danger of giving an overly optimistic impression of an intervention's effectiveness.

The most appropriate comparison is the most cost-effective alternative intervention currently available (the 'next best' alternative). Where the next best alternative is not clear cut, a number of alternatives may be considered, including the most widely practised alternative or current local practice (Byford 1998). Where there is little evidence of effectiveness or cost-effectiveness, a 'do nothing' alternative may be required, as it cannot be assumed that existing services are better than doing nothing.

\section{Perspective}

The perspective or analytical viewpoint assumed in an economic evaluation determines which costs and benefits are included in the analysis. A good economic evaluation should always explicitly state the perspective of the analysis. Analytical viewpoints commonly adopted in the UK include: patients and carers, the health and social care system, the government, and the broad societal perspective. The National Institute for Health and Care Excellence (NICE) guidelines currently mandate the patient and the NHS/personal social services (NHS/PSS) perspectives for benefits, but only NHS/PSS costs in submissions for approval of new health technologies (NICE 2013). Potential benefits for other governmental bodies, 
such as reductions in crime resulting from drug treatment programmes, may also be considered, if appropriate. Productivity losses, an important component of the wider societal perspective, are explicitly rejected, as these costs do not fall directly on NHS or government budgets.

The narrow perspective currently used by NICE is problematic when assessing the true costs and benefits of mental health services. For example, many healthcare evaluations do not include patient costs or the cost of informal care (care provided by friends or family), which may make community-based interventions appear unduly favourable compared with residential care. Box 1 lists the broad range of costs that may be relevant to users of mental health services. As can be seen, only the first three cost items are considered under current NICE health technology assessment guidelines (NICE 2013), despite the contributions made by other sectors. NICE does recognise the limitations of the narrow health and social care perspective with respect to costs, allowing for the inclusion of broader perspectives where considered appropriate, but

BOX 1 Resources relevant to users of mental health services

- Social care services (e.g. social work, accommodation, day care)

- Primary healthcare services (e.g. general practitioners, health visitors)

- Secondary healthcare services (e.g. psychiatric services, clinical psychology)

- Education services (e.g. educational psychologists, education welfare officers)

- Education facilities (e.g. schools for children with intellectual disabilities, pupil referral units)

- Voluntary-sector services (e.g. Childline, Barnardo's, Alcoholics Anonymous)

- Private-sector services (e.g. counselling, alternative therapies)

- Accommodation (e.g. sheltered housing, staffed hostels)

- Criminal justice (e.g. family courts, youth offending teams, probation, prisons)

- Patient and family costs (e.g. travel to services, child care)

- Unpaid informal care (e.g. care provided by family or friends)

- Productivity costs (e.g. time off work or unemployment because of disability)

(Adapted from Byford 2003) the inclusion of broader societal costs in economic evaluation is complex, can add considerably to the administrative and data requirements in an evaluation and can be contentious. For example, counting productivity losses may discriminate against patients with mental health problems with limited employment prospects.

\section{Costs}

\section{Identification}

Costs from the health economic perspective begin with the resource use implications of alternative treatments (i.e. the resources relevant to mental health summarised in Box 1). A good economic evaluation should provide a full description of the resources consumed or saved by competing interventions. This is important for the generalisability of findings, since readers can then revalue the resource use to suit their own settings (Drummond 2005). A simple approach to identifying relevant health inputs is to pose the question 'Who does what to whom, where and how often?' (Drummond 2005: p. 30). The type of services included will depend on the perspective of the study and on an understanding of patients and clinical pathways. The main methods of identifying relevant resource use include reviewing the literature, patient focus groups and clinical opinion. Useful sources of information to identify appropriate cost items include the Database of Instruments for Resource Use Measurement (DIRUM; www.dirum.org) (Ridyard 2012), the NHS Economic Evaluation Database (NHS EED; www.crd.york.ac.uk/crdweb) and the Health Technology Assessment (HTA) library (www. journalslibrary.nihr.ac.uk/hta).

\section{Measurement}

Once the resources relevant to an economic evaluation have been identified, they need to be measured and valued. Resource use is measured in discrete units such as the number of hours worked, sessions delivered and quantities of drugs or other consumables. For example, a course of psychotherapy can be measured in terms of the number of sessions or the duration of contact. Non-faceto-face time spent preparing for sessions, writing notes and liaising with other professionals should be measured in working hours, whereas training and supervision specific to the intervention could be measured in either sessions or working hours. Various methods are available to collect resource use data, including clinical records, patient or carer questionnaires and direct observation of clinical activity (Byford 2003). 
BOX 2 Outcomes relevant to economic evaluation of mental healthcare

- Patient and carer quality of life

- Mortality

- In-patient admission and length of stay

- Symptom relief

- Drug misuse

- Criminal justice contact (offending, incarceration)

- Treatment engagement

- Treatment satisfaction

- Medication adherence

- Employment status

- Educational attainment

- Carer burden

\section{Valuation}

The total cost of an intervention is the product of the quantity of each resource item used and the unit cost of each item (Petrou 2011a). Unit costs should include all the costs associated with the provision of the service being costed. These include, for example, clinical salaries, training costs and the cost of shared resources such as overheads, buildings, utilities and equipment. A good economic evaluation will report the same price year for all cost data, with adjustments using healthcare-specific inflation indices if needed (Petrou 2011a). In the UK, the Personal Social Services Research Unit (PSSRU) has published annual estimates of the unit costs of personal social services including mental healthcare each year since 2001 (e.g. Curtis 2014). This is a convenient and authoritative source of unit costs for mental healthcare in community and hospital settings across a range of treatment groups and modalities.

\section{Consequences}

Consequences from the health economic perspective are the final economic endpoints valued by patients, health services or society as

TABLE 1 Summary of methods of economic evaluation

\begin{tabular}{|lll|}
\hline Method & Costs & Effects \\
\hline $\begin{array}{l}\text { Cost-effectiveness analysis } \\
\text { (CEA) }\end{array}$ & Monetary values (e.g. f) & Single disease-specific outcome \\
\hline Cost-utility analysis (CUA) & Monetary values (e.g. f) & $\begin{array}{l}\text { Generic measure of quality of life/ } \\
\text { utility (e.g. to calculate 0ALYs) }\end{array}$ \\
\hline Cost-benefit analysis (CBA) & Monetary values (e.g. f) & Monetary values (e.g. f) \\
\hline $\begin{array}{l}\text { Cost-consequences analysis } \\
\text { (CCA) }\end{array}$ & Monetary values (e.g. f) & $\begin{array}{l}\text { Range of disease-specific } \\
\text { outcomes }\end{array}$ \\
\hline
\end{tabular}

QALY, quality-adjusted life-year. a whole. Consequences can also be called effects, outcomes, benefits or outputs, and these terms are often used interchangeably. Box 2 lists some outcomes relevant to the economic evaluation of mental healthcare services. Some of these, for example treatment engagement, are process or intermediate outcomes, whereas others, such as improving patient quality of life, are the ultimate aims of mental healthcare. Although outcomes will depend on the specific objectives and audience for an economic evaluation, it is patient benefit that is usually of most interest to economists and policy makers.

The quality-adjusted life-year (QALY) is a generic measure of health outcome that combines survival and quality of life. One QALY represents 1 year lived in full health. QALYs can be weighted to reflect the strength of preference for time spent in poor health compared with full health. Preference weightings for QALYs are estimated from surveys of the general public or of patient groups. The QALY is the preferred outcome measure for reimbursement and guideline development agencies such as NICE because it provides a common metric to compare different treatments and different conditions (NICE 2013).

QALYs are commonly derived from healthrelated quality of life outcome measures such as the EQ-5D (Brooks 1996), a generic measure of health-related quality of life preferred by NICE (2013). It is a brief outcome measure covering five health domains (mobility, usual activities, pain and discomfort, depression and anxiety, and selfcare). Some argue that generic measures of quality of life that focus on physical health may not be sensitive to the full range of impacts of complex interventions in mental health (Mulhern 2014).

\section{Methods of economic evaluation}

There are a number of methods of economic evaluation that can be used to compare the relative efficiency (or cost-effectiveness) of alternative mental healthcare interventions (Drummond 2005). All involve the identification, measurement, valuation and comparison of all relevant costs and consequences. All measure costs in monetary terms, for example pounds sterling or US dollars. They differ, however, in their approach to measuring consequences or benefits of interventions, and also in the questions they answer (Table 1).

\section{Cost-effectiveness analysis}

Cost-effectiveness analysis (CEA) involves the valuation of consequences, or effects, using a single condition- or service-specific outcome measure 
such as level of depression or life-years gained. The effects of two or more interventions are combined with their respective costs to provide a measure of cost-effectiveness that can be compared with other interventions using the same measures of effect. Box 3 shows how cost-effectiveness analysis has been used in mental health.

CEA does have its weaknesses. The singledimensional outcomes used in CEA are assumed to be the most worthwhile and appropriate, but most mental health programmes have an impact on multiple outcomes (e.g. social functioning, symptoms and carer burden). A further problem with CEA is comparability between different mental health programmes (e.g. dementia in the elderly and autism in children) and between mental health and other healthcare programmes (e.g. depression $v$. stroke). Healthcare programmes with different aims cannot be compared with each other using CEA. Thus, CEA is most useful when comparing programmes within similar areas that share common outcome measures.

\section{Cost-utility analysis}

Cost-utility analysis (CUA) is a form of CEA that enables broader, more generic comparisons between treatments for different diseases and conditions. Multidimensional health outcomes are measured by a single preference- or utility-based index such as the QALY. The main limitation, as indicated earlier, is the lack of health-related quality-of-life outcome scales with known validity and sensitivity in mental health populations. Box 4 shows how CUA has been used in mental health.

\section{Cost-benefit analysis}

Cost-benefit analysis (CBA) values all the consequences, or benefits, of an intervention in monetary terms. It is the predominant method of economic evaluation in many policy areas, including transport, health and safety, and the environment. $\mathrm{CBA}$ requires that all the consequences of an intervention, such as life-years saved, treatment side-effects, symptom relief, disability, pain and discomfort, are allocated a monetary value. It then becomes possible to directly compare the costs and benefits of an intervention, since both are expressed in the same monetary units. If the monetary value of the benefits exceeds the costs, then the intervention should be adopted subject to any overriding budgetary constraint. CBA asks whether an intervention is worthwhile. Unlike in CEA and CUA, which ask which interventions should be adopted to maximise health, a comparator is not needed in a CBA, although the net benefit of competing interventions can be

BOX 3 Example from the literature: cost-effectiveness analysis of an intervention for psychosis

Randomised controlled trial of joint crisis plans to reduce compulsory treatment for people with psychosis: economic outcomes (Barrett 2013)

This study examined the cost-effectiveness of joint crisis plans (JCPs) compared with treatment as usual (TAU) for patients with a history of psychiatric admission. JCPS are developed by patients together with mental health staff. They include patient treatment preferences for future psychiatric emergencies when the patient may be too unwell to express them. Data were collected from the services perspective (health, social and criminal justice services) and the societal perspective (criminal activity, employment) for 504 patients.
JCP was not significantly more effective than TAU in terms of compulsory admissions and had a low probability of being more cost-effective from the societal perspective. However, from the services perspective, which is the most relevant to public-sector policy makers, JCP had a high probability of being more cost-effective.

This is also an illustration of how small non-significant differences in costly and distressing events such as compulsory psychiatric admissions can still be costeffective. The authors acknowledged that the lack of a generic quality-of-life instrument was a study limitation.

compared. Box 5 shows how CBA has been used in mental health.

The obvious problem with CBA is that it is very difficult to convert benefits from mental health programmes into monetary values. Concerns about placing a monetary value on human life and suffering were among the founding arguments in support of CEA as the preferred evaluative framework for healthcare interventions in place of CBA (Gold 1996). Other health economists argue that techniques now exist to elicit such values, and that ignoring values that individuals and patients place on health benefits leads to an inefficient allocation of resources (McIntosh 2010). Nevertheless, the methods of CBA are difficult to apply and can be a time-consuming and costly addition to an evaluation. Consequently, CBA

BOX 4 Example from the literature: cost-utility analysis of treatments for chronic heroin addiction

Cost-effectiveness of injectable opioid treatment $v$. oral methadone for chronic heroin addiction (Byford 2013)

This study compared the cost-effectiveness of supervised injectable opioid treatment (heroin or methadone) with that of oral methadone in 127 patients with treatmentrefractory addiction who continued to use illicit heroin. Data were collected on use of clinic resources, other health and social care resources and criminal justice resources, and quality-adjusted life-years (QALYS) calculated using EQ-5D-derived utilities.
The clinical resources needed to provide injectable opioid treatment were significantly more costly than those required for oral methadone, but methadone was the most costly option after factoring in the costs of criminal activity. QALY gains were greater in the injectable opioid groups compared with the oral methadone group. Thus, in the cost-utility analysis, oral methadone was dominated by injectable opioids, being more expensive and less effective. 
BOX 5 Example from the literature: cost-benefit analysis of government policy on cannabis

Cost-benefit analysis of two policy options for cannabis: status quo and legalisation (Shanahan 2014)

This study used an economic model to value the costs and benefits of two cannabis policy options in Australia - the status quo where cannabis is illegal, and an alternative where cannabis is legalised and regulated.

Monetary values were placed on: the stigma of a criminal record; reduced income due to lost educational attainment; the value of well-being from cannabis consumption; lost wages due to imprisonment; value of lives lost in motor vehicle accidents; costs of law enforcement (policing, courts, prisons); personal costs to cannabis users (lost employment, fines); cannabis-related healthcare costs (cannabis use disorder, schizophrenia and psychosis, low birthweight babies); and in addition for the legalisation and regulation option, the cost of the regulatory system. A range of economic techniques were used to value the model inputs, including, for example, a survey of 875 people to estimate their willingness to pay to avoid the stigma of a criminal record.

The net social benefit (NSB) of each policy was calculated by subtracting the costs from the benefits. After estimating percentiles around the mean point estimates, the authors concluded that there was no difference in the NSB for either policy option, i.e. neither was more economically favourable than the other.

is relatively rarely used in mental health service evaluation. Some studies described as CBAs take a limited approach to the valuation of outcomes, focusing only on measures of outcome that can easily be costed, for example cost savings as a result of reductions in criminal activity. However, these are more appropriately termed cost-offset studies since they do not attempt to value the full benefit to patients.

\section{Cost-consequences analysis}

Cost-consequences analysis (CCA) involves the presentation of a range of outcome measures

BOX 6 Example from the literature: cost-consequences analysis of mental health nurse prescribing

A comparison of the clinical effectiveness and costs of mental health nurse supplementary prescribing and independent medical prescribing: a post-test control group study (Norman 2010)

This study compared the cost and clinical impact of mental health supplementary prescribing with those of medical prescribing in 90 patients matched for age, gender, primary diagnosis and time since diagnosis. The cost of mental health nurse prescribing included the time costs of training, supervision and consultation. Data were also collected from patients on their use of health and social care services, and unpaid informal care from family and friends. Outcome data included medication adherence, medication satisfaction depression, social functioning, patientperceived health improvement, medication adverse effects and patient satisfaction.

This was the first study to investigate the effectiveness and costs of mental health nurse prescribing and so the analyses were exploratory. Thus, a cost-consequences analysis was undertaken where costs and multiple outcomes were summarised by group but not brought together. The authors reported that there were no significant differences in either patient outcomes or costs between the two sets of prescribers. alongside costs. No attempt is made to formally combine costs with outcomes, and decision makers are left to form their own opinion regarding the relative importance of the alternative outcomes presented (Mauskopf 1998). CCA has been recommended for complex interventions that may have an impact on multiple areas of an individual's life, such as social, psychological and family functioning, that can be difficult to measure in a common unit of outcome (Drummond 2005). CCAs are not restricted to any viewpoint, so policy makers can see the impact of their decisions for patients in different settings or other sectors such as criminal justice (Brazier 2007). Box 6 shows how CCA has been used in mental healthcare. The main drawback of a CCA is that it does not rank interventions by cost-effectiveness or give any definitive guidance on cost-effectiveness thresholds.

\section{Combined approaches}

The approaches described above are not necessarily mutually exclusive. A study can conduct a CEA based on the primary clinical outcome to facilitate comparisons within disease areas and also conduct a CUA to generalise findings. The presentation of a CCA can enhance the understanding gained from an economic evaluation even when a primary outcome has been selected and a CEA or CUA has been carried out.

\section{Interpretation}

To determine the relative cost-effectiveness of alternative interventions, and hence inform policy decisions, rules are needed to enable decision makers to clearly understand under what circumstances a service can be considered more cost-effective than an alternative. Decision rules in traditional cost-benefit analysis are relatively straightforward because benefits and costs are both measured in monetary terms. Thus, when the monetary value of the benefits exceeds the monetary value of the costs, the intervention should be funded, subject to existing budgetary constraints.

Combining costs with outcomes in CEA or CUA is more complex. The decision rule is based on two quantities: the additional monetary cost of a new treatment compared with an existing alternative, and the additional benefits measured in terms of health gains. This can be expressed as the incremental cost-effectiveness ratio (ICER) (Van Hout 1994). The ICER is the difference in costs divided by the difference in effects:

$$
\text { ICER }=\left(C_{\mathrm{n}}-C_{\mathrm{c}}\right) /\left(E_{\mathrm{n}}-E_{\mathrm{c}}\right)
$$


where $C_{\mathrm{n}}$ is the cost of the new service; $C_{\mathrm{c}}$ is the cost of the comparison group; $E_{\mathrm{n}}$ is the effectiveness of new service; $E_{\mathrm{c}}$ is the effectiveness of the comparison group.

The ICER can be plotted on the cost-effectiveness plane, as in Fig. 1. On this graph, the origin $(0,0)$ represents the comparison group. The change in costs and effects due to the new intervention relative to the comparison group can be plotted on the plane. The four quadrants have different policy implications. In the south-east quadrant, the new intervention is more effective and less costly and should be adopted. It is said to dominate the existing strategy. In the north-west quadrant, the new intervention is more costly and less effective and should be rejected. In this case, the new intervention is dominated by the existing strategy. If the new intervention turns out to be more effective but more costly (north-east quadrant) or less effective but less costly (south-west quadrant), trade-offs need to be made: either the additional cost of the new intervention is justified by its additional benefits (north-east quadrant) or the reduction in effectiveness is justified by the costs saved (south-west quadrant).

When the results of an evaluation involve a tradeoff between costs and effects, further information is needed to determine whether the savings or additional benefits are justified. To answer this question, health economists use the concept of willingness to pay, which is the maximum amount a decision maker is willing to pay for a unit improvement in effects (usually denoted by the Greek symbol lambda $\lambda$ ). Any services that have an ICER below the ceiling ratio $(\lambda)$ should be funded, within the constraints of existing budgets. In the UK, NICE has set this maximum value with respect to QALY gains at $£ 20000$ to $£ 30000$ or less, although there is controversy about how this threshold value is set (see Claxton 2015).

\section{Uncertainty}

Economic evaluation involves two major sources of uncertainty: where an intervention is located on the cost-effectiveness plane and how much a decision maker is willing to pay for health gains (Petrou 2011a). However, since the ICER is a ratio, producing confidence intervals is not straightforward. For example, ICERs in the northwest quadrant (more costly, less effective) have the same negative sign as ICERs in the south-east quadrant (less costly, more effective) but have diametrically opposed interpretations.

One solution to this problem of uncertainty around the ICER is the cost-effectiveness acceptability curve (CEAC) (Fenwick 2005).

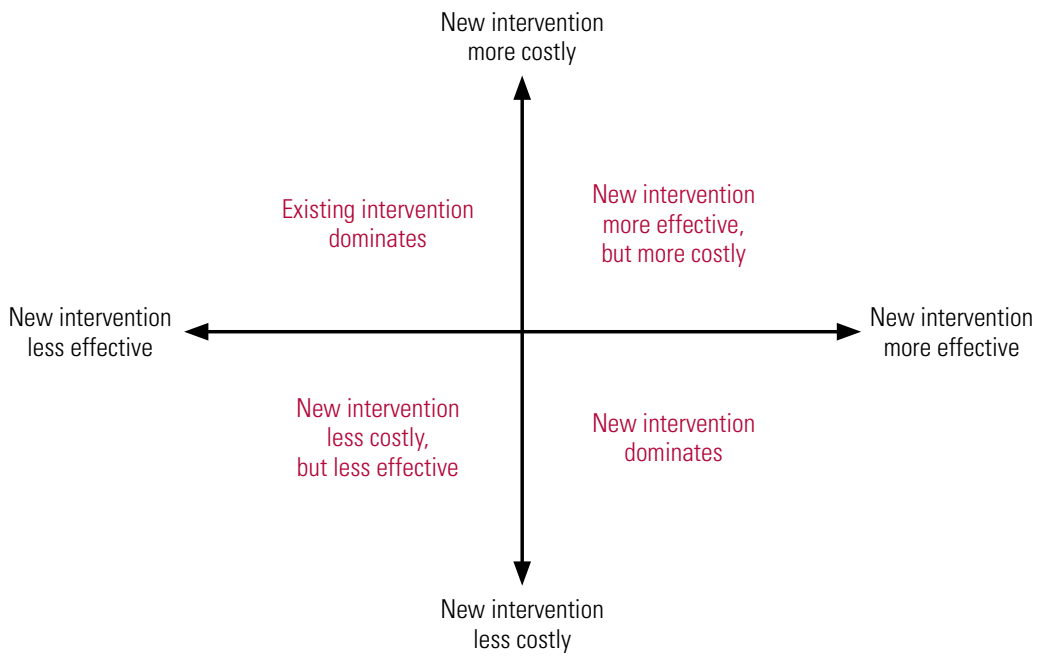

FIG 1 A cost-effectiveness plane for alternative interventions.

CEACs are derived from the joint distribution of the difference in costs and the difference in effects. The joint distribution is often generated using nonparametric bootstrapping of the observed data, which produces a scatter plot of incremental cost and effect pairs on the cost-effectiveness plane.

Example scatter plots for the societal and services perspectives of the study outlined in Box 3 appear in Fig. 2 (note that outcomes are reversed compared with Fig. 1, which is the more typical presentation). The proportion of points falling to the south and east of a line drawn through the origin with a slope equal to the ceiling ratio $\lambda$ represents the probability that the intervention is cost-effective for that level of willingness to pay. The CEAC plots the probability that the intervention is cost-effective for a range of values of $\lambda$. The CEACs for the societal and services perspectives in Box 3 appear in Fig. 3. The CEAC from the services perspective shows that the probability of joint crisis plans (JCPs) being more cost-effective than treatment as usual (TAU) was at least $80 \%$ for every value of willingness to pay, including zero. However, from the societal perspective (which includes the costs of crimes and lost employment) the probability that JCP was more cost-effective than TAU was lower, at $44 \%$, but increased as willingness to pay increased, rising to over $50 \%$ at $£ 9000$ and above.

\section{Longer-term extrapolation}

Economic modelling is used to extrapolate costs, consequences and disease progression beyond the time frame of RCTs, particularly when the benefits occur long after an intervention, as for example the benefits of early diagnosis or smoking cessation. Decision analytic modelling synthesises data from multiple sources, including RCTs, 


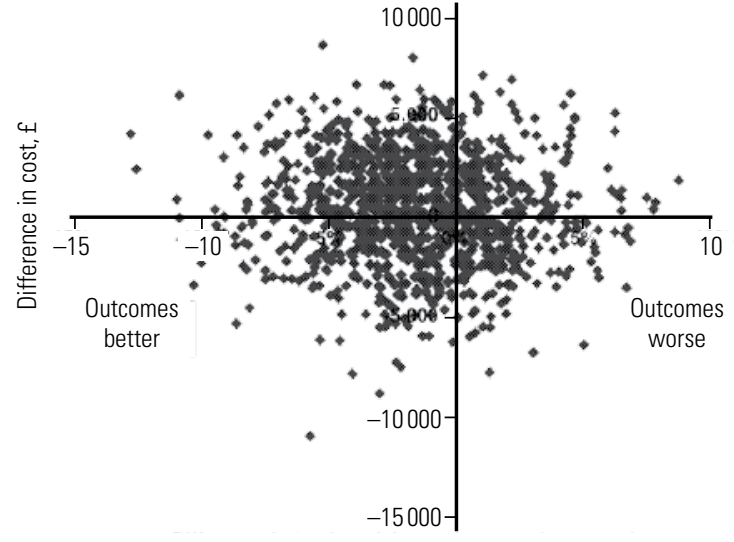

(a) Difference in \% of participants in compulsory section

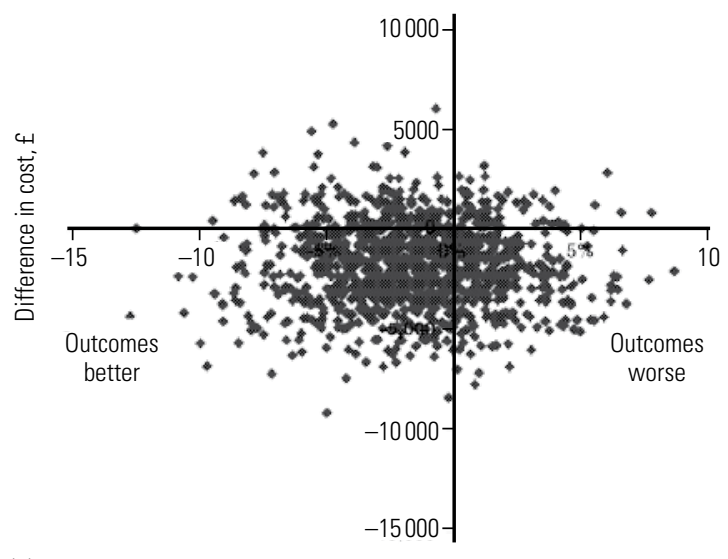

(b) Difference in \% of participants in compulsory section

A cost-effectiveness plane: bootstrapped cost and effectiveness pairs for (a) societal costs and (b) service costs (Barrett 2013, with permission).

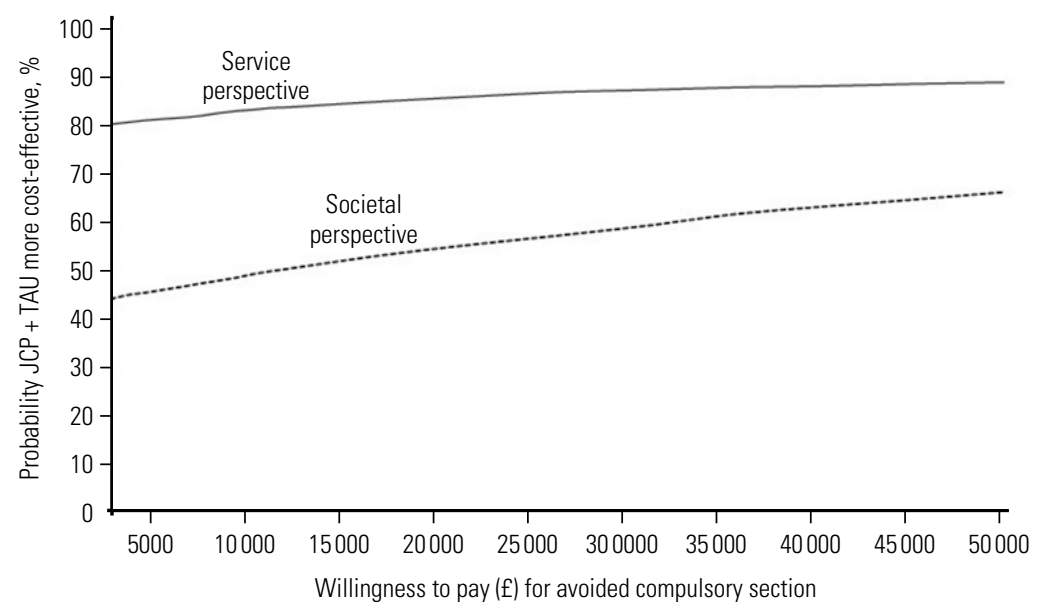

A cost-effectiveness acceptability curve showing the probability that joint crisis plans plus treatment as usual (JCP+TAU) is more cost-effective than TAU over 18-month followup (Barrett 2013, with permission).

to simulate costs and outcomes over different time horizons, clinical settings or treatment MCQ answers 1 d 2 c 3 e 4 a 5 c populations (Briggs 2006; Petrou 2011b). The value of future costs and outcomes is discounted to reflect a time preference for deferred costs and earlier health gains. This is because people value the same benefit more highly if it is received today rather than at some time in the future. Similarly, people value money spent today more highly than the same amount spent some time in the future. The discount rate acts much like an interest rate which calculates the present value of future costs and benefits. Modelling introduces extra layers of complexity that can be confusing or unfamiliar to a clinical audience (and to many health economists!). These include advanced mathematical and computational techniques used to simulate outcomes and estimate the uncertainty inherent when combing data from disparate sources. However, modelling has become essential in decision-making and NICE health technology decisions are based on economic models rather than the evidence from a single trial.

\section{Critical appraisal of economic evaluations}

A number of checklists are available for assessing the quality of published economic evaluations and their relevance to specific decision-making contexts (e.g. see Drummond 1996). The NHS Economic Evaluation Database mentioned earlier (www.crd.york.ac.uk/crdweb) provides critical reviews of economic evaluations published up to and including 2014.

\section{References}

Barrett B, Waheed W, Farrelly S, et al (2013) Randomised controlled trial of joint crisis plans to reduce compulsory treatment for people with psychosis: economic outcomes. PLOS ONE, 8(11): e74210.

Brazier J, Ratcliffe J, Salomon J, et al (2007) Measuring and Valuing Health Benefits for Economic Evaluation. Oxford University Press.

Briggs A, Claxton, K, Sculpher M (2006) Decision Modelling for Health Economic Evaluation. Oxford University Press.

Brooks R (1996) Euro0ol: the current state of play. Health Policy, 37: 53-72.

Byford S, Palmer S (1998) Common errors and controversies in pharmacoeconomic analyses. PharmacoEconomics, 13: 659-66.

Byford S, McDaid D, Sefton T (2003) Because It's Worth It: A Practical Guide to Conducting Economic Evaluations in the Social Welfare Field. Joseph Rowntree Foundation.

Byford S, Barrett B (2010) Ethics and economics: the case for mental heathcare. Advances in Psychiatric Treatment, 16: 468-73.

Byford S, Barrett B, Metrebian N, et al (2013) Cost-effectiveness of injectable opioid treatment $v$. oral methadone for chronic heroin addiction. British Journal of Psychiatry, 203: 341-9.

Claxton K, Sculpher M, Palmer S, et al (2015) Causes for concern: is NICE failing to uphold its responsibilities to all NHS patients? Health Economics, 24: 1-7.

Curtis L (ed) (2014) PSSRU Unit Costs of Health and Social Care 2014. Personal Social Services Research Unit.

Drummond M, Jefferson T (1996) Guidelines for authors and peer reviewers of economic submissions to the BMJ. BMJ, 313: 275-83.

Drummond M, Sculpher M, Torrance G, et al (2005) Methods for the Economic Evaluation of Health Care Programmes. Oxford University Press.

Fenwick E, Byford S (2005) A guide to cost-effectiveness acceptability curves. British Journal of Psychiatry, 187: 106-8. 
Fineberg NA, Haddad PM, Carpenter L, et al (2013) The size, burden and cost of disorders of the brain in the UK. Journal of Psychopharmacology, 27: $761-70$.

Gold M, Siegel J, Russell L, et al (1996) Cost-Effectiveness in Health and Medicine. Oxford University Press.

Guiness L, Wiseman V (2011) Introduction to Health Economics (2nd edn). Open University Press

Jacobs R (2014) Payment by results for mental health services: economic considerations of case-mix funding. Advances in Psychiatric Treatment, 20: $155-64$.

Mauskopf J, Paul J, Grant D, et al (1998) The role of cost-consequences analysis in healthcare decision-making. Pharmacoeconomics, 13: 277-88.

McCrone PR, Dhanasiri S, Patel A, et al (2008) Paying the Price: The Cost of Mental Health Care in England to 2026. King's Fund.

McIntosh E, Clarke P, Frew E, et al (2010) Applied Methods of CostBenefit Analysis in Health Care. Oxford University Press.

Mulhern B, Mukuria C, Barkham M, et al (2014) Using generic preferencebased measures in mental health: psychometric validity of the EQ-5D and SF-6D. British Journal of Psychiatry, 205: 236-43.
National Institute for Health and Care Excellence (2013) Guide to the Methods of Technology Appraisal 2013 (Process and Methods Guides PMG9). NICE.

Norman I, Coster S, McCrone P, et al (2010) A comparison of the clinical effectiveness and costs of mental health nurse supplementary prescribing and independent medical prescribing: a post-test control group study. BMC Health Services Research, 10(4).

Petrou S, Gray A (2011a) Economic evaluation alongside randomised controlled trials: design, conduct, analysis, and reporting. BMJ, 342: d1548.

Petrou S, Gray A (2011b) Economic evaluation using decision analytical modelling: design, conduct, analysis, and reporting. BMJ, 342: d1766.

Ridyard CH, Hughes DA (2012) Development of a database of instruments for resource-use measurement: purpose, feasibility, and design. Value in Health, 15: 650-5

Robinson R (1993) Economic evaluation and health care: what does it mean? BMJ, 307: 670-3.

Shanahan M, Ritter A (2014) Cost-benefit analysis of two policy options for cannabis: status quo and legalisation. PLoS ONE, 9(4): e95569.

Van Hout BA, Al MJ, Gordon GS, et al (1994) Costs, effects and C/E-ratios alongside a clinical trial. Health Economics, 3: 309-19.

\section{MCOs}

Select the single best option for each question stem

1 A treatment is cost-effective when:

$\mathrm{a}$ it is cheaper than the alternatives

$\mathrm{b}$ it is cheap

c it is standard recommended practice

$\mathrm{d}$ it is cheaper and more effective than the alternatives

e it is more effective than the alternatives.

\section{Opportunity cost is:}

a the financial cost of a treatment

b the resources consumed by a treatment

c the foregone benefits of the next best use of resources

$d$ the savings from disinvesting in an ineffective treatment e the savings from more efficient use of current resources.

3 Costs relevant to the health and social care system perspective include:

a costs of criminal activity

b employment losses

c informal unpaid care by family and friends

d special education services

e none of the above.

\section{Effectiveness is measured on the basis of} differences in quality-adjusted life-years by:

a cost-utility analysis

b cost-benefit analysis

c cost-effectiveness analysis

d cost-consequences analysis

e all of the above.
5 An intervention is said to be dominant if:

a it is cheap

$b$ it has a large effect size

c it is cheaper and more effective than alternatives

$\mathrm{d}$ it is cheaper and less effective than alternatives

e it is more costly and more effective than alternatives. 